\title{
Quantum computation with trapped polar molecules
}

\author{
D. DeMille \\ Department of Physics, P.O. Box 208120, Yale University, New Haven, CT 06520
}

(Dated: October 27, 2001)

\begin{abstract}
We propose a novel physical realization of a quantum computer. The qubits are electric dipole moments of ultracold diatomic molecules, oriented along or against an external electric field. Individual molecules are held in a 1-D trap array, with an electric field gradient allowing spectroscopic addressing of each site. Bits are coupled via the electric dipole-dipole interaction. Using technologies similar to those already demonstrated, this design can plausibly lead to a quantum computer with $\gtrsim 10^{4}$ qubits, which can perform $\sim 10^{5}$ CNOT gates in the anticipated decoherence time of $\sim 5 \mathrm{~s}$.

PACS numbers: 03.67.Lx, 33.80.Ps, 33.55.Be
\end{abstract}

We describe a new technical approach to the design of a quantum computer (QC). The basic QC architecture is shown in Fig. 1. The qubits consist of the electric dipole moments of diatomic molecules, oriented along or against an external electric field. Bits are coupled by the electric dipole-dipole interaction. Individual molecules are held in a 1-D trap array, with an electric field gradient allowing spectroscopic addressing of each site. Loading with ultracold molecules makes it possible to use a weak trapping potential, which should allow long decoherence times for the system. This design bears various features in common with other recent proposals which employ electric dipole couplings [1, 2, 3]. However, the technical parameters of our design appear very favorable, and apparently only incremental improvements of demonstrated techniques are required in order to build a $\mathrm{QC}$ of unprecedented size.

We describe the molecular qubits as permanent electric dipoles oriented along $(|0\rangle)$ or against $(|1\rangle)$ an external electric field $\left(\vec{E}_{e x t}\right)$. (This model reproduces the exact behavior well in a certain regime.) Lattice sites are equally spaced in the x-direction and each contains one molecule, prepared initially in its ground state $|0\rangle$. The external field is perpendicular to the trap axis and

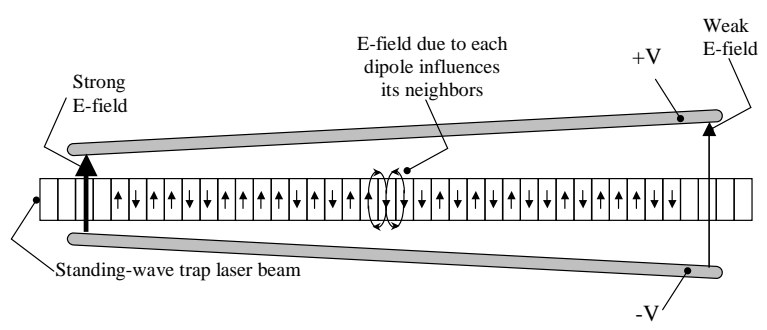

FIG. 1: Schematic depiction of the polar molecule quantum computer. Molecules are trapped in a 1-D optical lattice. Qubit states correspond to electric dipole moments up or down relative to the applied E-field. A field gradient makes the resonant frequency for each qubit unique. The electric field of each dipole changes the energy of its neighbors, according to their relative orientations. consists of a constant bias field plus a linear gradient: $\vec{E}_{\text {ext }}(x)=\left[E_{0}+x(\partial E / \partial x)\right] \hat{z}$. The Hamiltonian for bit $a$ at position $x_{a}$ is $H_{a}^{\prime}=H^{0}-\vec{d}_{a} \cdot \vec{E}_{a}$, where $H^{0}$ is the internal energy of a bit, $\vec{d}_{a}$ is the electric dipole moment of bit $a$, and $\vec{E}_{a}=\vec{E}_{e x t}\left(x_{a}\right)+\vec{E}_{i n t}\left(x_{a}\right)$ is the total electric field at $x_{a}$. The internal field $\vec{E}_{\text {int }}$ is created by the electric dipole moments of neighboring bits: $\vec{E}_{i n t}\left(x_{a}\right)=\sum_{b \neq a} \frac{-\vec{d}_{b}}{\left|x_{a}-x_{b}\right|^{3}}$. For reasonable operating parameters, $E_{\text {ext }} \gg E_{\text {int }}$.

The scheme for gate operations is as outlined for the electric dipole moments of quantum dots in Ref. [1]. Transitions between qubit states can be driven by electric resonance, either directly in the microwave region or indirectly by an optical stimulated Raman process. Resonant drive pulses are tuned to frequency $\nu_{a}=\nu_{0}+d_{e f f} E_{a} / h$, where $h \nu_{0}$ is the difference in internal energies between states $|0\rangle$ and $|1\rangle$ in zero field; the effective dipole moment $d_{e f f}=\left|\vec{d}_{|0\rangle}-\vec{d}_{|1\rangle}\right|$, where $\vec{d}_{|0\rangle(|1\rangle)}$ is the dipole moment in state $|0\rangle(|1\rangle)$; and $h$ is Planck's constant. Pulses of sufficient temporal length to resolve the energy splitting due to $E_{\text {int }}$ can be used for CNOT gates; shorter pulses suffice for one-bit rotations. Final-state readout can be accomplished by state-selective, resonant multiphoton ioniza-

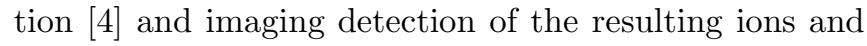
electrons.

The efficient creation of ultracold diatomic molecules by photoassociation of laser cooled atoms was recently demonstrated [5, 6, 7, 8]. Electronically excited neutral molecules are produced by a laser-induced transition from the free state of two atoms; the excited state can subsequently decay into bound vibrational levels of the molecular ground state. The molecules are formed at a translational temperature similar to that of the constituent atoms; $T \approx 20 \mu \mathrm{K}$ has been demonstrated [7].

Production of ultracold atoms is most advanced for alkali atoms. Fortunately, heteronuclear bi-alkali molecules are well suited to our purposes. While no such species have yet been produced at ultracold temperatures, there seems to be no fundamental obstacle to making them. The rate-limiting Franck-Condon (FC) factors in the formation process in general should be more favorable for 
hetero- than for homo-nuclear species, because of the better match between ground- and excited-state potential curves [9, 10]. Homonuclear bi-alkalis $\mathrm{K}_{2}, \mathrm{Rb}_{2}$, and $\mathrm{Cs}_{2}$ have been formed, as well as heteronuclear molecular ions $\mathrm{NaCs}^{+}$[11]. Molecules formed by photoassociation are typically in the lowest rotational states $(J=0-2)$, but spread over many vibrational levels $(v)$. High vibrational levels $(v>100)$ of $\mathrm{Cs}_{2}$ were formed at a total rate of $>10^{6} / \mathrm{s}$ [5, 7]; in a more complex scheme, $\mathrm{K}_{2}$ molecules were produced at rates of up to $10^{5} / \mathrm{s} /$ level, in low vibrational states $(v \sim 10)[6]$. Based on the calculated FC factors and the demonstrated production of homonuclear species, a production rate of $\gtrsim 10^{5} / \mathrm{s}$ ultracold heteronuclear molecules in individual rovibrational levels seems feasible. Molecules in any state with $J=0$ or 2 and $v \gg 1$ can be transferred efficiently to the ground state $(v=0, J=0)$ via a stimulated Raman transition [12].

For the bi-alkali molecules, there is some tradeoff between ease of production and the size of the molecular dipole moment. The FC factors for photoassociation are largest for pairs of atoms with similar excitation energies [9], while the dipole moments are largest for pairs where these are most different [13]. We specifically consider the KCs molecule, which has both a moderately large dipole moment and substantial FC factors; however, the other bi-alkali species have similar properties and one of them might prove ultimately more favorable.

An optical trap appears to be suitable for creating the desired 1-D array of molecules. For laser frequencies detuned to the red of any electronic transition, the dynamic polarizability gives rise to a force that attracts both atoms and molecules [14] to regions of high intensity. Far off-resonance traps are weak, but extremely non-perturbative [15]. Such traps are well developed for atoms, with demonstrated trap lifetimes $\gtrsim 300 \mathrm{~s} \mid 16$, and internal state decoherence times $\gtrsim 4 \mathrm{~s} \mathrm{[17].} \mathrm{Trapping}$ of molecules in an off-resonant laser beam was recently demonstrated for ultracold $\mathrm{Cs}_{2}$ [18].

Our proposed trap consists of a 1-D optical lattice, superposed with a crossed dipole trap [19] of cylindrically focused beams. This confines the molecules in sites spaced by $\lambda_{t} / 2$ (where $\lambda_{t}$ is the trap laser wavelength). The molecules will be well localized in these wells for trap depth $U_{0} \gg k T$; we assume $U_{0}=100 \mu \mathrm{K}$ is sufficient. We take $\lambda_{t} \sim 1 \mu \mathrm{m}$ as a convenient compromise between small trap spacing and increased decoherence rates. For a homogeneous trap of length $L$, we require that the Rayleigh length $z_{0}=\pi \omega_{0}^{2} / \lambda_{t}>L$, where $\omega_{0}$ is the beam waist. We take $L=5 \mathrm{~mm}\left(\sim 10^{4}\right.$ trap sites $)$ and $\omega_{0}=50 \mu \mathrm{m}$. Transverse confinement is determined by the cylindrical beam waist $\omega_{t}$; we assume diffractionlimited beams with $\mathrm{f} / 1$ focusing to achieve $\omega_{t} \sim \lambda_{t}$.

For given $\lambda_{t}$ and laser power, the trap depth is determined by the KCs dynamic polarizability, which is not known in detail. However, it is possible to crudely estimate the required parameters. For moderate laser fre- quency detuning $\Delta$, the polarizability will be dominated by the oscillator strength of the first excited ${ }^{1} \Sigma$ level, which should couple to the ground state with a transition dipole moment comparable to that for the $6 s-6 p$ transition of Cs 20]. For $\Delta \gg \omega_{e}$ (the molecular vibrational frequency), the FC structure is irrelevant. Thus, for the same detuning the trap depth for KCs should be similar to that for atomic Cs. We find that $\Delta \approx 2000 \mathrm{~cm}^{-1}$ gives reasonable behavior. For KCs this corresponds to a trap wavelength $\lambda_{t} \approx 1.1 \mu \mathrm{m}$, and requires only $\approx 1 \mathrm{~W}$ of laser power for the 1-D lattice (as for Cs [21]). The cross-sectional area of each transverse beam is $\sim 2 \times$ that of the 1-D lattice beam, so the power in these must be comparable to achieve transverse confinement to $\sim \lambda_{t} / 2$. The required lasers are commercially available.

$\mathrm{K}$ and $\mathrm{Cs}$ atoms can be loaded into such an optical trap from a standard magneto-optic trap. If necessary, the temperature of the atoms can be reduced in the trap by a variety of methods polarization gradient cooling 19 , 22, 23. The two-species sample in this trap should have $N \gtrsim 10^{7}$ atoms with density $n \gtrsim 10^{11} \mathrm{~cm}^{-3}$ and $T \lesssim$ $20 \mu \mathrm{K}$. Photoassociation for $\sim 1 \mathrm{~s}$ and stimulated Raman transfer should produce $\sim 10^{5}$ molecules in the ground molecular state. Remaining atoms (vibrationally-excited molecules) can be removed from the trap by resonant light pushing (selective photoionization [10]).

Remarkably, it may prove relatively easy to distribute the remaining molecules such that exactly one populates each lattice site. It has been argued that the repulsive interaction between atoms in a Bose condensate can lead to a Mott insulator-like phase transition, and thus unity filling of an optical lattice 24]. The interactions between polarized molecules are many orders of magnitude stronger than for atoms, and thus may facilitate reaching a similar phase transition even without Bose condensation. Detailed calculations are necessary to confirm this speculation, which does not take into account the anisotropy of the dipole-dipole interaction [25]. The large collision cross-sections for the polarized molecules [26] should also make it possible to achieve fast rates of evaporative cooling, and thus (if necessary) an even lower temperature than that of the original constituent atoms; the molecules can be held in their ground state in this phase, to avoid losses due to inelastic collisions. We note in passing that the final molecular temperature and density $\left(n \sim\left(2 / \lambda_{t}\right)^{3} \sim 10^{13} \mathrm{~cm}^{-3}\right)$ discussed here correspond to a phase-space density of $\sim 10^{-3}$, far from Bose condensation.

In the absence of an external field, even polar molecules have no net electric dipole moment. The application of an external field mixes rotational states; for low fields the mixed state which arises from the $J=0\left(J=1, m_{J}=0\right)$ state corresponds to a dipolar charge distribution along (against) $\vec{E}_{\text {ext }}$. Calculations of the effect of $E_{\text {ext }}$ on these two states are shown in Fig. 2. The energies for $E_{\text {ext }}=0$ are $E_{J}=h B J(J+1)$, where the rotational constant $B \approx$ 

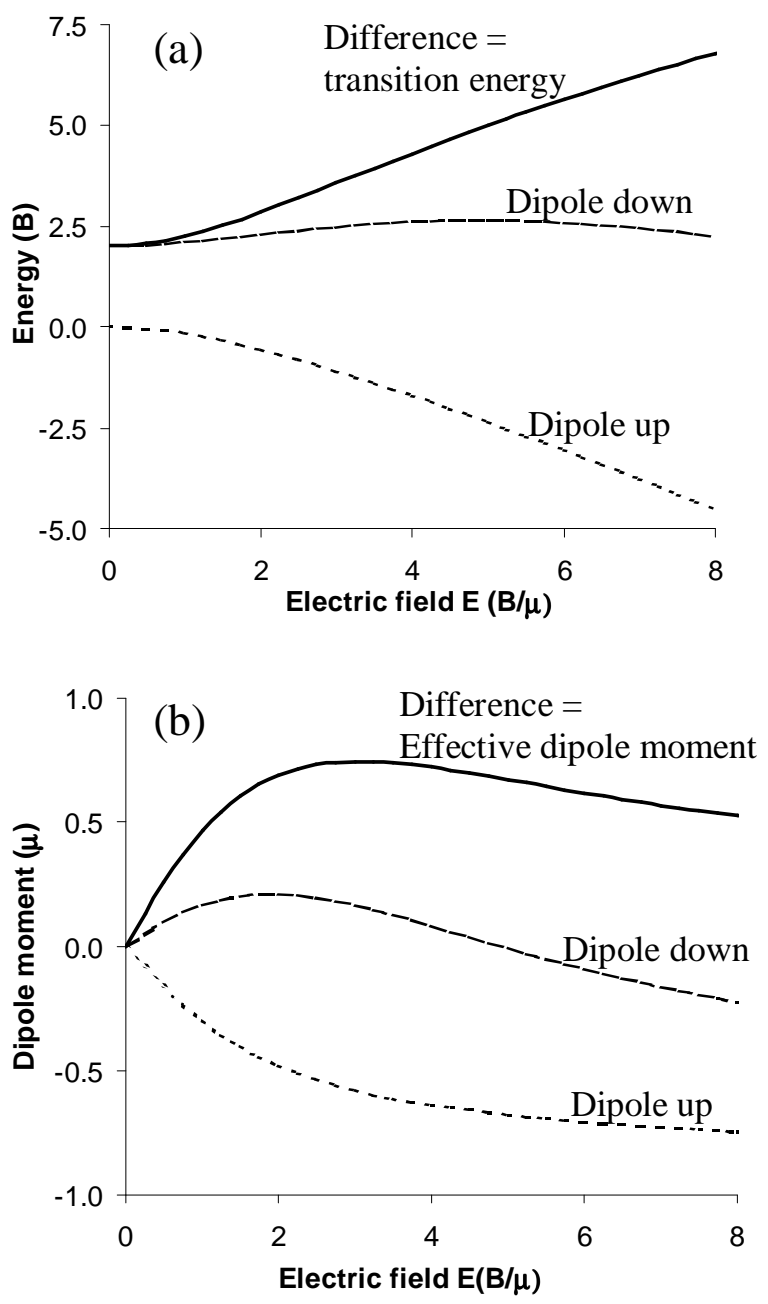

FIG. 2: Effect of an electric field on a polar molecule. a) Energy levels. b) Induced dipole moments.

$1.0 \mathrm{GHz}$ for KCs 13]. Stark matrix elements are taken from standard formulae [27], using the calculated value of the molecule-fixed dipole moment for KCs, $\mu=1.92 \mathrm{D}$ [13]. In order to perform CNOT gates, it is necessary to resolve the transitions $|0\rangle|0\rangle \Leftrightarrow|0\rangle|1\rangle$ from $|1\rangle|0\rangle \Leftrightarrow$ $|1\rangle|1\rangle$. These differ in energy by $h \delta \nu=d_{\text {eff }}^{2} /\left(\lambda_{t} / 2\right)^{3}$. Over a wide range of electric field strengths $E_{\text {ext }}=(2-$ $5) B / \mu(\approx 2-5 \mathrm{kV} / \mathrm{cm}$ for $\mathrm{KCs}), d_{\text {eff }}$ is within $10 \%$ of its maximum value $(0.75 \mu)$. The time required for CNOT gates is $\tau \gtrsim(2 \pi \delta \nu)^{-1} \approx 50 \mu$ s. The one-bit drive frequencies $\nu_{a}$ cover the range $3.5-6.0 \mathrm{GHz}$ over the array, with approximately equal steps of $250 \mathrm{kHz}$ between sites. Direct microwave drive of a CNOT gate requires rf electric field strength $\approx 10 \mathrm{mV} / \mathrm{cm}$ for a $\pi$-pulse.

The final state of the register can be read out by rapidly (but adiabatically) turning off $\vec{E}_{\text {ext }}$, then applying a laser pulse to perform resonantly-enhanced multiphoton ionization [20]. Commercial pulsed lasers with $\sim$ ns pulse widths have both sufficient energy for $\sim 100 \%$ ionization efficiency $(\sim \mathrm{mJ} /$ pulse $)$, and sufficient spectral resolution $(\ll 2 B \approx 2 \mathrm{GHz})$ to make contamination from the undesired logic state negligible. Molecules in each state can be detected by consecutive identical laser pulses, with an intervening rf $\pi$-pulse to transfer population between logic states. Simple ion optics can magnify the ionized array image 10-fold, so that the charges form a pattern $5 \mathrm{~cm}$ long, with spacing between ions of $5 \mu \mathrm{m}$. The magnified charge array can be detected on an imaging microchannel plate. Commercial detectors are available with sufficient size and resolution; detection of both ions and electrons from each logic state should lead to effective efficiencies $\gtrsim 90 \%$.

The most important known source of decoherence is photon scattering from the trap laser. The total offresonance photon scattering rate is dominated by inelastic (Raman) scattering to other rotational and vibrational levels 28]. For the chosen value of $\Delta$, the scattering rate for KCs should be comparable to the elastic scattering rate $R_{s}$ for Cs (much as for the trap depth). For the trap parameters discussed, $R_{s} \sim 0.2 \mathrm{~s}^{-1}$ [21].

We have considered several technical noise issues, all of which appear controllable at the desired level. The trap laser shifts the values of $\nu_{a}$, through coupling to the tensor polarizability of the molecule. Tensor shifts are typically several times smaller than the scalar shifts $\left(U_{0} \approx 2 \mathrm{MHz}\right)$ responsible for the trapping potential 114; we conservatively assume a tensor shift as large as $U_{0}$. We require that the 1-bit drive frequency have noise $\delta \nu_{a} \lesssim \sqrt{R_{s}} \sim 0.5 \mathrm{~Hz} / \sqrt{\mathrm{Hz}}$ [29]; this implies laser intensity stability $\delta I / I \lesssim 3 \times 10^{-7} / \sqrt{\mathrm{Hz}}$. This is $\sim 300 \times$ the shot-noise limit, and should be achievable [30]. Electric field noise couples directly to the molecular dipole moments, and is also of concern. With field plate spacing of $\sim 1 \mathrm{~cm}$, we require broadband voltage noise $\delta V \lesssim 0.5 \mu \mathrm{V} / \sqrt{\mathrm{Hz}}$, the room-temperature Johnson noise on a $10 \mathrm{M} \Omega$ resistor. Noise from the high-voltage supply can be heavily filtered and should pose no problems. A variety of other possible decoherence sources seem to present no limitations. These include heating due to laser intensity, beam-pointing, or frequency fluctuations [16, 31]; dissociation of molecules by the trap laser [32]; spontaneous emission; coupling to blackbody radiation; collisions with background gas molecules; etc.

We have shown that a quantum computer based on ultracold KCs molecules can plausibly achieve $\sim 10^{5}$ CNOT gates on $\sim 10^{4}$ bits in the anticipated decoherence time of $\sim 5 \mathrm{~s}$. This may be sufficient for quantum error correction methods to ensure that arbitrarily long computations are stable [33]. We have also argued that this system requires no dramatic technical breakthroughs for its initial construction. The electric resonance techniques for the processor should be robust and easy to implement, by analogy with similar NMR methods. Creation of the trapped array of polar molecules appears to be a direct extension of recent work in laser cooling and trap- 
ping, and the readout via resonance-enhanced ionization is standard. Unlike recent proposals for quantum computation using ultracold atoms, our technique requires neither mechanical motion [2, 34], nor coupling to shortlived excited states [2, 35], for gate operations.

There are a number of potentially serious issues that we have not considered. For example, the bit-bit interaction cannot be switched off, and thus operation will require techniques similar to the "refocusing" procedure used to control the couplings in NMR quantum computation [36]. We have ignored the motional states of the molecules; although the trap motional frequencies $(\sim 100 \mathrm{kHz})$ are well-separated from other frequency scales in the device, couplings of gate operations to the motion may cause additional decoherence or gate fidelity loss [35]. We have also ignored the hyperfine structure of the KCs molecules, which might complicate the initial state selection and/or gate operations. We plan to investigate these issues in the future. In the meantime, we have begun an experimental effort to implement these ideas (using RbCs rather than KCs for technical convenience).

On the other hand, the parameters discussed here might also be improved with other techniques that are currently less well developed. For example, buffer-gas cooling 37] or electric slowing and trapping [38], in combination with evaporative cooling 39], could yield larger and/or colder samples; the variety of molecules accessible to these techniques could enable the use of larger values of $\mu$ and/or smaller values of $\lambda_{t}$. Microfabricated traps based on low-frequency electromagnetic fields might prove advantageous [40], and non-destructive readout may be possible by direct pickup of the molecular dipole fields with nearby single-electron transistors 41 . Finally, in addition to our qubit states, there are $\sim 10^{6}$ long-lived rovibrational states available for each molecule 20]; these might allow each molecule to function as a quantum information unit containing $n \gg 1$ bits of information. Although entanglement between individual molecules is more difficult in this case, the massive parallelism involved may be useful in itself 42].

We thank M. Kasevich, P. Zoller, and A.J. Kerman for useful discussions. DD is an Alfred P. Sloan Research Fellow and a Packard Foundation Fellow. This work is supported by NSF ITR grant \#EIA-0081332.

[1] A. Barenco et al., Phys. Rev. Lett. 74, 4083 (1995).

[2] G. Brennen et al., Phys. Rev. Lett. 82, 1060 (1999).

[3] P. Platzman and M. Dykman, Science 284, 1967 (1999).

[4] W. Demtröder, Laser Spectroscopy, 2nd Ed. (SpringerVerlag, Berlin, 1996).

[5] Fioretti et al., Phys. Rev. Lett. 80, 4402 (1998).

[6] A. Nikolov et al., Phys. Rev. Lett. 84, 246 (2000).

[7] C. Drag et al., IEEE J. Quant. El. 36, 1378 (2000).

[8] C. Gabbanini et al., Phys. Rev. Lett. 84, 2814 (2000).
[9] H. Wang and W. Stwalley, J. Chem. Phys. 108, 5767 (1998).

[10] C. M. Dion et al., Phys. Rev. Lett. 86, 2253 (2001).

[11] J. Shaffer, W. Chalupczak, and N. Bigelow, Phys. Rev. Lett. 82, 6 (1999).

[12] See e.g. U. Gaubatz et al., J. Chem. Phys. 92, 5363 (1990)

[13] G. Igel-Mann et al., J. Chem. Phys. 84, 5007 (1986).

[14] B. Friedrich and D. Herschbach, Phys. Rev. Lett. 74, 4623 (1995); see also P. Braun and A. Petelin, Sov. Phys. JETP 39, 775 (1974).

[15] J. Miller, R. Cline, and D. Heinzen, Phys. Rev. A 47, R4567 (1993).

[16] K. O'Hara et al., Phys. Rev. Lett. 82, 4204 (1999).

[17] N. Davidson et al., Phys. Rev. Lett. 74, 1311 (1995).

[18] T. Takekoshi, B. Patterson, and R. Knize, Phys. Rev. Lett. 81, 5105 (1998).

[19] C. Adams et al., Phys. Rev. Lett. 74, 3577 (1995).

[20] Data on the typical electronic structure of bi-alkali molecules is taken from: A.A. Radzig and B.M. Smirnov, Reference Data on Atoms, Molecules, and Ions (Springer-Verlag, Berlin, 1980); K.P. Huber and G. Herzberg, Molecular Spectra and Molecular Structure: IV. Constants of Diatomic Molecules (Van Nostrand, New York, 1979); M. Korek et al., Can. J. Phys. 78, 977 (2000); A. Allouche et al., J. Phys. B 33, 2307 (2000); S. Magnier, M. Aubert-Frécon, and Ph. Millié, J. Mol. Spect. 200, 96 (2000); and references therein.

[21] M.V. Romalis and E.N. Fortson, Phys. Rev. A 59, 4547 (1999).

[22] S. Winoto et al., Phys. Rev. A 59, R19 (1999).

[23] See e.g. S. Hamann et al., Phys. Rev. Lett. 80, 4149 (1998).

[24] D. Jaksch et al., Phys. Rev. Lett. 81, 3108 (1998).

[25] L. Santos et al., Phys. Rev. Lett. 85, 1791 (2000).

[26] J.L. Bohn, Phys. Rev. A 63, 052714 (2001).

[27] C. H. Townes and A. L. Schawlow, Microwave Spectroscopy, (McGraw-Hill, New York, 1955).

[28] R. Loudon, The Quantum Theory of Light, 2nd Ed. (Clarendon, Oxford, 1983).

[29] S. Lamoreaux, Phys. Rev. A 56, 4970 (1997).

[30] J.L. Hall, in International Conference on Quantum Optics, ed. J. Harvey and D. Walls (Springer-Verlag, Berlin, 1986).

[31] T. Savard, K. O'Hara, and J. Thomas, Phys. Rev. A 56, R1095 (1997).

[32] G. Askar'yan, Sov. Phys. JETP 21, 439 (1965); V. Khersonskii, Opt. Spctrosc. (USSR) 43, 19 (1977).

[33] J. Preskill, in Introduction to Quantum Computation and Information, ed. H.-K. Lo, S. Posescu, and T. Spiller (World Scientific, Singapore, 1998).

[34] D. Jaksch et al., Phys. Rev. Lett. 82, 1975 (1999).

[35] D. Jaksch et al., Phys. Rev. Lett. 85, 2208 (2000).

[36] M.A. Nielsen and I.L. Chuang, Quantum Computation and Quantum Information (Cambridge Univ. Press, Cambridge, 2000).

[37] J.D. Weinstein et al., Nature 395, 148 (1998).

[38] H.L. Bethlem et al., Nature 406, 491 (2000).

[39] J. M. Doyle et al., Phys. Rev. A 52, R2515 (1995).

[40] T. Calarco et al., Phys. Rev. A 61, 022304 (2000).

[41] R.J. Schoelkopf et al., Science 280, 1238 (1998); R. Schoelkopf, private communication (2001).

[42] J. Ahn, T. Weinacht, and P. Bucksbaum, Science 287, 463 (2000); P. Knight, Science 287, 441 (2000). 